\title{
IL-10 Induction from Implants Delivering Pancreatic Islets and Hyaluronan
}

\author{
Paul L. Bollyky, ${ }^{1}$ Robert B. Vernon, ${ }^{2}$ Ben A. Falk, ${ }^{1}$ Anton Preisinger, ${ }^{2}$ Michel D. Gooden, ${ }^{2}$ \\ Gerald T. Nepom, ${ }^{2}$ and John A. Gebe ${ }^{2}$ \\ ${ }^{1}$ Division of Infectious Diseases and Geographic Medicine, Department of Medicine, Stanford University School of Medicine, \\ Grant Building, 300 Pasteur Drive, Stanford, CA 94305-5107, USA \\ ${ }^{2}$ Benaroya Research Institute, 1201 Ninth Avenue, Seattle, WA 98101-2795, USA
}

Correspondence should be addressed to John A. Gebe; jgebe@benaroyaresearch.org

Received 5 February 2013; Revised 8 June 2013; Accepted 13 June 2013

Academic Editor: Shahidul Islam

Copyright (C) 2013 Paul L. Bollyky et al. This is an open access article distributed under the Creative Commons Attribution License, which permits unrestricted use, distribution, and reproduction in any medium, provided the original work is properly cited.

Local induction of pro-tolerogenic cytokines, such as IL-10, is an appealing strategy to help facilitate transplantation of islets and other tissues. Here, we describe a pair of implantable devices that capitalize on our recent finding that hyaluronan (HA) promotes IL-10 production by activated T cells. The first device is an injectable hydrogel made of crosslinked HA and heparan sulfate loaded with anti-CD3/anti-CD28 antibodies and IL-2. T cells embedded within this hydrogel prior to polymerization go on to produce IL-10 in vivo. The second device is a bioengineered implant consisting of a polyvinyl alcohol sponge scaffold, supportive collagen hydrogel, and alginate spheres mediating sustained release of HA in fluid form. Pancreatic islets that expressed ovalbumin (OVA) antigen were implanted within this device for 14 days into immunodeficient mice that received OVA-specific DO.11.10 T cells and a subsequent immunization with OVA peptide. Splenocytes harvested from these mice produced IL-10 upon re-challenge with OVA or anti-CD3 antibodies. Both of these devices represent model systems that will be used, in future studies, to further evaluate IL-10 induction by HA, with the objective of improving the survival and function of transplanted islets in the setting of autoimmune (type 1) diabetes.

\section{Introduction}

Interleukin 10 (IL-10) is a potent immunosuppressive cytokine made by regulatory $\mathrm{T}$ cells (Tregs) and other cell types [1-3]. IL-10 inhibits antigen-specific immune responses in part via suppression of activated macrophage and monocyte functions, which include cytokine synthesis and expression of class II MHC and costimulatory molecules such as IL-12 and CD80/CD86 [4].

IL-10 has important roles in transplant biology. Endogenous IL-10 production is correlated with transplant acceptance in multiple animal models and human tissues [58]. IL-10 has been evaluated as a treatment to improve the survival of engrafted islets, which has been accomplished by transfer of IL-10-producing Tregs [6], gene therapy $[9,10]$ or direct administration of IL-10 alone, or in conjunction with immunomodulatory drugs [11-13]. It is noteworthy that systemic IL-10 treatment has failed to support islet engraftment in mice in the setting of established autoimmunity [14] and may induce immune suppression. These results suggest that an alternative approach that provides a sustained, local presence of IL-10 at the graft site might be more effective at preventing islet rejection.

We recently reported a role for the extracellular matrix (ECM) macromolecule hyaluronan (HA) in regulating IL10 production by $\mathrm{T}$ cells. $\mathrm{HA}$ is a simple, long-chain glycosaminoglycan polymer made up of repeating disaccharides of $\mathrm{N}$-acetyl glucosamine and glucuronic acid. HA is an important structural component of many tissues, but also has important roles in inflammation and tissue repair [15-18]. Short HA oligomers $(<30 \mathrm{kDa})$ generated through tissue catabolism are typically proinflammatory [16-18]. Conversely, plate-bound HA or chemically crosslinked HA is antiinflammatory and promotes IL-10 production by FoxP3(+) 
natural Tregs (nTregs) [19] and conventional T cells in vitro [20]. Induction of IL-10 in these systems was mediated by crosslinking of CD44, the primary receptor for HA [20]. We have proposed that plate-bound HA and HA hydrogels may function as biomimetics of HA-containing tissue matrices. However, the minimum size for HA-mediated CD44 crosslinking and IL-10 production by $\mathrm{T}$ cells is unknown. Additional support for a role for HA in IL-10 production is provided by observations of HA-induced upregulation of IL10 by cultured synoviocytes [21] and elevated IL-10 levels in intestinal biopsies of mice given oral HA [22]. However, HA alone does not appear to promote IL-10 induction by T cells in vitro. Indeed, our data suggest that concomitant antigenic stimulation through the $\mathrm{T}$ cell receptor (TCR) complex is required for efficient IL-10 induction in the presence of HA.

HA preparations are currently used as treatments for arthritis [23], atopic dermatitis [24], prevention of abdominal adhesions $[25,26]$ and are under evaluation as an experimental treatment for burns and wounds [27, 28]. In most of these preparations, $\mathrm{HA}$ is crosslinked to promote its stability and efficacy [29]. Crosslinking (as well as platebinding or sustained release from alginate) may also limit the generation of pro-inflammatory HA fragments. Building upon these findings, we have evaluated whether HA has utility in promoting IL-10 production in vivo.

Here, we describe and evaluate a pair of technologies that both provide antigenic stimulation in the context of HA. First, we have asked whether cells implanted within a crosslinked HA hydrogel that incorporates a supplemental complex to induce polyclonal TCR stimulation could enhance production of IL-10 in vivo. Second, we have developed a bioengineered implant capable of delivering an antigenic signal along with sustained release of HA in fluid form. These technologies represent parallel strategies for delivering HA as a medium to promote IL-10 production in vivo, with the ultimate objective of inducing durable immune tolerance to transplanted islets in individuals with autoimmune diabetes.

\section{Materials and Methods}

2.1. Transgenic Mice. C57BL/6 green fluorescent protein (GFP)-FoxP3 knock-in and RIPmOVA/Rag2 $2^{-/-}$mice were the kind gifts of Dr. A. Rudensky (Memorial Sloan-Kettering Cancer Center, New York, NY, USA) and Dr. Steve Ziegler (Benaroya Research Institute-BRI), respectively. DO11.10 mice were purchased from Taconic Farms. All mice were maintained in a specific pathogen-free, AAALAC-accredited facility at BRI, and all experiments were approved by the BRI Institutional Animal Care and Use Committee (IACUC), protocol approval number 10116.

2.2. Isolation of Leukocyte Populations. Mouse lymphocyte populations were prepared as previously described [19]. In brief, for the in vitro experiments, CD4(+) cells were isolated using MACS kits (Miltenyi, Inc.), and the GFP-FoxP3(-) fraction was isolated from the $\mathrm{CD} 4(+)$ population using a FACS Vantage cell-sorter (BD Biosciences). CD4(+)/GFPFoxP3(-) $\mathrm{T}$ cells were used to ensure that any IL-10 production we measured would be from conventional $\mathrm{T}$ cells, rather than from activated GFP-FoxP3(+) nTregs. Cells were cultured in Opti-MEM (Invitrogen) serum-free media supplemented with $100 \mu \mathrm{g} / \mathrm{mL}$ penicillin and $100 \mathrm{U} / \mathrm{mL}$ streptomycin $(\mathrm{P} / \mathrm{S})$. Where specifically noted, cells were cultured in complete media consisting of Dulbecco's Modified Eagle's Medium (DMEM)-10 (Invitrogen) supplemented with $10 \%$ fetal bovine serum (FBS) (Hyclone), $\mathrm{P} / \mathrm{S}, 50 \mu \mathrm{M} \beta$ mercaptoethanol, $2 \mathrm{mM}$ glutamine, and $1 \mathrm{mM} \mathrm{Na}$ pyruvate (Invitrogen).

\subsection{In Vitro T Cell Activation Using Plate-Bound Antibodies} and HA. Cell culture plates (96-well) were coated with $0.5 \mu \mathrm{g} / \mathrm{mL}$ of anti-CD3 antibody (145-2C11, BD Biosciences), washed, and then subsequently coated with either $0.2 \mathrm{mg} / \mathrm{mL}$ bovine serum albumin (BSA)- conjugated HA $\left(1.5 \times 10^{6} \mathrm{Da}\right)$ HA (Genzyme) or 10\% BSA. CD4(+)/GFP-FoxP3(-) T cells $\left(2 \times 10^{5}\right.$ per well) were cultured for 96 hours on these plates, followed by collection of the culture supernatants for analysis. Measurement of cytokines in the cell culture supernatants was performed using enzyme-linked immunosorbent assays (ELISAs) or cytometric bead assays (BD Biosciences).

2.4. In Vitro T Cell Activation Using HA Hydrogels. Hydrogels were made from thiolated constituents (HA, heparin sulfate [HS], and collagen) crosslinked with polyethylene glycol $\mathrm{S}-\mathrm{S}$ diacrylate (PEGSSDA). These reagents are available as a kit (Extracel-HP, Glycosan/Biotime) and were used per the manufacturer's instructions. Of note, our understanding from communications with the manufacturer is that HA of $>1 \times 10^{6} \mathrm{Da}$ is used in the kits. Prior to addition of the crosslinker, the mixture was supplemented with $10 \mu \mathrm{g} / \mathrm{mL}$ of streptavidin (Sigma Aldrich), $10 \mu \mathrm{g} / \mathrm{mL}$ each of biotinylated anti-CD3 and anti-CD28 antibodies (145-2C11, 37.51, BD Biosciences), and $20 \mathrm{IU} / \mathrm{mL}$ of IL-2. Hydrogels of this formulation are referred to here as "supplemented HA hydrogels." For in vitro cell culture experiments, $2 \times 10^{5} \mathrm{CD} 4(+) / \mathrm{GFP}-$ FoxP3(-) T cells were layered on top of $25 \mu \mathrm{L}$ volumes of the hydrogel. After 96 hours of culture, cells and culture supernates were collected for analysis. To control the collagen constituent of the HA hydrogels, a set of hydrogels lacking $\mathrm{HA} / \mathrm{HS}$ was made by replacing the thiolated HA/HS with an equivalent volume of thiolated collagen. These controls are referred to as "supplemented collagen hydrogels."

2.5. Implantation of T Cells and HA. $3 \times 10^{6} \mathrm{CD} 4(+) / \mathrm{GFP}-$ FoxP3(-) T cells were dispersed in supplemented HA hydrogels of $300 \mu \mathrm{L}$ volume prior to crosslinking with PEGSSDA, which was initiated $30 \mathrm{~min}$ prior to intraperitoneal injection into mice. For these studies in vivo, the supplemented HA hydrogels incorporated $360 \mathrm{IU} / \mathrm{mL}$ of IL-2. Four days after injection of the supplemented HA hydrogels, the mice were sacrificed and lymphoid tissues were harvested. Residual hydrogel material in the peritoneal cavity was also removed and dissolved by mild reduction of the PEGSSDA (per the manufacturer's instructions) in order to retrieve cells for analysis. Intracellular staining of these cells for IL-10 and subsequent flow cytometry assays utilized antibodies and equipment as previously described [19]. 
2.6. Isolation of Islets. Islets were isolated as described previously [30]. Briefly, C57Bl/6 mice of 12-24 weeks age were anesthetized with 2,2,2-tribromoethanol in phosphatebuffered saline (PBS). The descending aorta of each anesthetized mouse was transected, the bile duct clamped at its distal (intestinal) end, and a 30-gauge needle was used to inflate each pancreas through the common bile duct with $4 \mathrm{~mL}$ of $4^{\circ} \mathrm{C}$ Islet Medium comprised of RPMI 1640 containing $1.0 \mathrm{~g} \mathrm{NaHCO}_{3}, 10 \%$ FBS (Atlanta Biologicals, cat. number $\mathrm{S} 12450 \mathrm{H}), 1 \mathrm{mM}$ Na-pyruvate, and P/S. The Islet Medium was supplemented with $0.8 \mathrm{mg} / \mathrm{mL}$ of collagenase $\mathrm{P}$ (Roche, cat. number 11-249-002-001) and filtered at $0.22 \mu \mathrm{m}$ prior to injection. Subsequently, 2-3 excised pancreata were placed in separate $50 \mathrm{~mL}$ conical centrifuge tubes and incubated in $5 \mathrm{~mL}$ of Islet Medium for $13 \mathrm{~min}$ at $37^{\circ} \mathrm{C}$. The medium was then decanted, fresh $4^{\circ} \mathrm{C}$ Islet Medium was added, and the tubes were shaken vigorously to disrupt the pancreata. The tissue suspensions were filtered through a 30 -mesh metal screen to remove large debris, the filtrates were pelleted by centrifugation, and the pellets resuspended in $4^{\circ} \mathrm{C}$ Islet Medium. The resuspended material was centrifuged through Histopaque 1077 to isolate the islets, which were washed, resuspended in Islet Medium, and placed in a tissue culture (TC) incubator. After all pancreata were processed, the isolated islets were hand picked, cultured overnight, and picked again the next day before being placed in bioengineered implants. Average yields were $100-150$ islets per mouse.

\subsection{Fabrication of Bioengineered Implants (BIs)}

2.7.1. Polyvinyl Alcohol (PVA) Scaffolds. Biopsy punches (Sklar Instruments) were used to cut $10 \mathrm{~mm}$ diameter disks from $2 \mathrm{~mm}$ thick sheets of PVA sponge (Type CF90, $500 \mu \mathrm{m}$ average pore size with no surfactant treatment-a generous gift from Merocel/Medtronic, Inc.). Subsequently, each disk was through-punched with a single central hole of $2 \mathrm{~mm}$ diameter and six peripheral holes of $1.5 \mathrm{~mm}$ diameter, using correspondingly sized biopsy punches (Acuderm, Inc.). The punched disks were washed $5 \times 10 \mathrm{~min}$ on a rocker in $50 \mathrm{~mL}$ centrifuge tubes filled with $40 \mathrm{~mL}$ of sterile distilled water, then air-dried on Whatman filter paper, transferred to $60 \mathrm{~mm}$ dishes, sterilized by gamma irradiation, and stored until needed for BI assembly.

2.7.2. Type I Collagen Solution. One volume of a stock solution of rat tail native type I collagen in dilute $(0.02 \mathrm{~N})$ acetic acid (BD Biosciences) was combined with $1 / 9$ volume of 10-strength $\mathrm{NaHCO}_{3}$-saturated Medium 199 (Invitrogen) and sufficient DMEM and normal mouse serum (NMS) to yield a working solution containing $2.5 \mathrm{mg} / \mathrm{mL}$ collagen and $10 \%$ NMS [30]. The working solution was prepared just prior to assembly of the BIs and kept on ice until needed.

2.7.3. Alginate Spheres. An aqueous stock solution of $4 \%$ alginate (Sigma-Aldrich, cat. number A0682), filtered at $0.45 \mu \mathrm{m}$, was used for preparation of spheres for sustained release of vascular endothelial growth factor (VEGF) and HA. Spheres incorporating human recombinant $\mathrm{VEGF}_{165}$ (Peprotech, cat. number 100-20) were prepared as described previously [30]. Briefly, a mixture of $2 \%$ alginate and $5 \mathrm{ng} / \mu \mathrm{L}$ VEGF was formed into $10 \mu \mathrm{L}$ (2.2 mm diameter) spheres using a gravitydrop method, crosslinked into a hydrogel for $15 \mathrm{~min}$ in $0.1 \mathrm{M} \mathrm{CaCl}_{2}$, washed $2 \times 2 \mathrm{~min}$ in $0.15 \mathrm{M} \mathrm{NaCl} / 25 \mathrm{mM}$ $\mathrm{HEPES} / 2 \mathrm{mM} \mathrm{CaCl}_{2}, \mathrm{pH} 7.2$ (saline/HEPES/Ca), transferred to serum-free $\mathrm{DMEM} / \mathrm{P} / \mathrm{S}$, and kept in a tissue culture incubator until needed for BI assembly.

Fabrication of HA spheres was similar to that of the VEGF constructs, with replacement of the VEGF with $50 \mu \mathrm{g}$ of $120 \mathrm{kDa}$ HA (Genzyme). HA of this size (approximately 317 disaccharide units) was chosen to facilitate a complete delivery of HA from the spheres within a 2 week experimental time period.

2.7.4. Assembly of BIs. Dry PVA sponge scaffolds were allowed to swell for $5 \mathrm{~min}$ in sterile DMEM/P/S. Subsequently, a single, freshly prepared alginate sphere containing VEGF and five spheres containing HA were gently pressed into the 6 peripheral holes of each expanded scaffold. The scaffolds were then blotted on sterile Whatman filter paper, transferred to $60 \mathrm{~mm}$ plastic cell culture dishes lined with UV-sterilized Parafilm M, and flooded with $60 \mu \mathrm{L}$ of type I collagen working solution containing suspended islets. The PVA sponges absorbed the collagen solution, with the majority of the islets entering the $2 \mathrm{~mm}$ diameter central hole of the scaffold. Subsequently, the dishes were covered with dish tops (lined with moist filter paper) and placed in a tissue culture incubator for $30 \mathrm{~min}$ to polymerize the collagen into a hydrogel. The completed BIs were placed in DMEM/10\% NMS/P/S in 24-well cell culture plates and kept in a tissue culture incubator prior to implantation in mice.

2.7.5. Measurement of Release of HA from Alginate Spheres In Vitro. To measure the kinetics of release of HA from alginate hydrogels in vitro, spheres containing $2 \%$ alginate and $2.5 \mu \mathrm{g}$ of fluorescein isothiocyanate (FITC)-conjugated $120 \mathrm{kDa}$ HA or $1.5 \times 10^{6} \mathrm{Da} \mathrm{HA}$ were prepared as described previously. The spheres were placed in 96-well cell culture plates (one sphere per well) that had each of the wells filled with $200 \mu \mathrm{L}$ of Dulbecco's Ca/Mg PBS (DPBS Ca/Mg, Invitrogen). The plates were placed in a tissue culture incubator, and $100 \mu \mathrm{L}$ volumes of the media were removed from each well at specific time points (up to 14 days) and analyzed by fluorescence spectrophotometry to quantify released FITC-HA, using a standard curve of fluorescence versus known concentration of FITC-HA. Following removal of the medium at each time point, the residual medium in each well was discarded, and each well was refilled with $200 \mu \mathrm{L}$ of fresh medium.

To determine the percentage of HA retained in alginate spheres during their fabrication, freshly prepared spheres containing $2.5 \mu \mathrm{g}$ of FITC-HA were dissolved in PBS/ $100 \mathrm{mM}$ ethylenediaminetetraacetic acid (EDTA), and the resultant solution was analyzed by fluorescence spectrophotometry to quantify total FITC-HA per sphere.

2.7.6. Implantation of BIs In Vivo. BIs were implanted into mesenteric pockets of RIPmOVA/Rag2 ${ }^{-/-}$mice (one BI per 


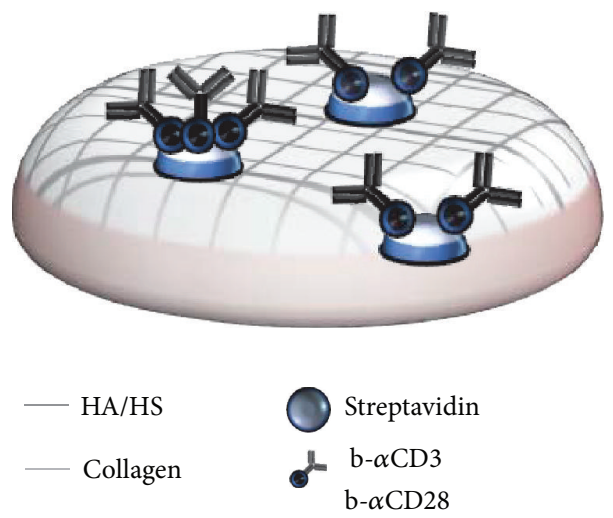

(a)

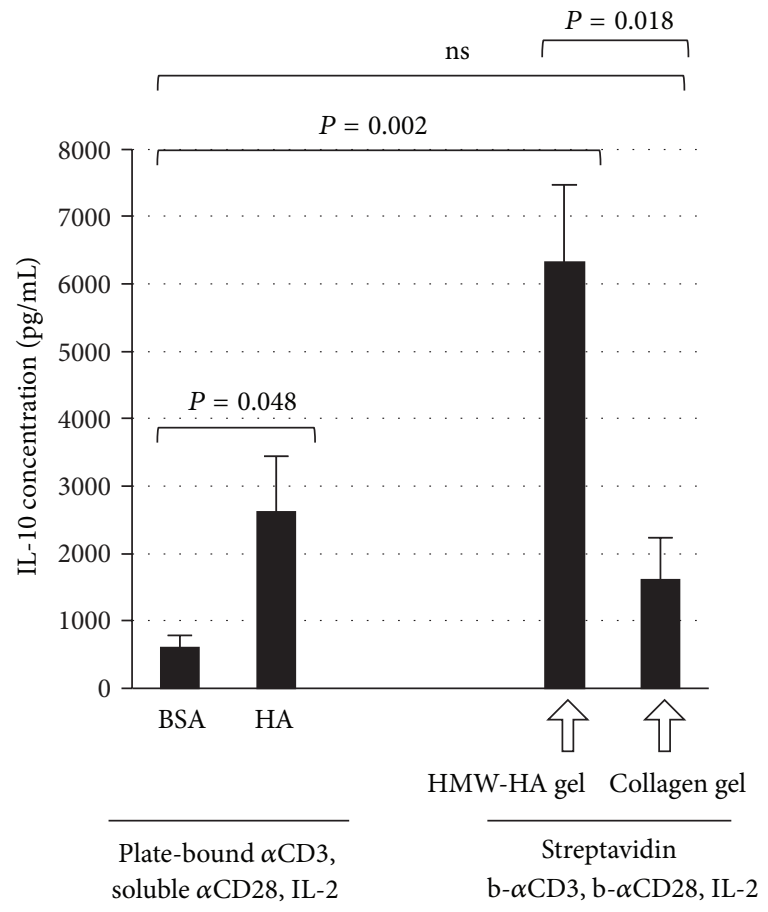

(b)

FIGURE 1: Induction of IL-10 by supplemented HA hydrogels in vitro. (a) Schematic for the design of a hydrogel capable of delivering a TCR stimulus in association with an HA signal. The "supplemented HA hydrogel" incorporates a crosslinked matrix of thiolated high molecular weight (HMW)-HA, HS, and collagen to which we have added streptavidin and biotinylated anti-CD3 and anti-CD28 antibodies (b- $\alpha \mathrm{CD} 3$, b- $\alpha$ CD28) to deliver an activating signal through the TCR. (b) Concentration of IL-10 in supernates taken from T cell cultures 96 hours after either plate-based (left side of graph) or hydrogel-based (right side of graph) activation (cells were cultured on top of the hydrogels for the latter experiments). Supplemented collagen hydrogels that lacked HA were used as a negative control. $n=5$ independent experiments.

mouse) using previously described protocols [30], followed by injection of the mice 24 hours later with $1 \times 10^{5}$ purified OVA-specific CD4(+) DO11.10 T cells.

2.8. Statistical Analyses. Statistical comparison of samples was made using Student's $t$-test.

\section{Results}

3.1. Supplemented HA Hydrogels Promote IL-10 Production In Vitro. We previously demonstrated that plate-bound HA together with an antigenic signal promotes IL-10 production by CD4(+)/GFP-FoxP3(-) T cells. This led us to ask whether we could develop this finding into a tool for use in promoting IL-10 production in vivo.

To this end, we modified a HA-based hydrogel to deliver a polyclonal antigenic stimulus through addition of streptavidin, biotinylated anti-CD3/CD28 antibodies, and IL-2. A schematic of this hydrogel design is shown in Figure 1(a). We have previously shown that a similar form of supplemented HA hydrogel is an efficient way to elicit IL-10 production from $\mathrm{T}$ cells in vitro [20].

We found that CD4(+)/GFP-FoxP3(-) T cells exposed to the supplemented HA hydrogels produced IL-10 at significantly higher levels than did corresponding $\mathrm{T}$ cells activated with anti-CD3/CD28 antibodies and IL-2 on cell culture plates (Figure 1(b)). This was the case whether the cells were cultured on top of the gels (as shown) or embedded within the gels (data not shown). Omission of either streptavidin or anti-CD3 antibody from the gel mixture likewise abrogated IL-10 production (data not shown), indicating that CD3 was required for the stimulus and suggesting that streptavidin was necessary to retain $\mathrm{CD} 3$ in the hydrogel lattice. Streptavidin, biotinylated anti-CD3/CD28 antibodies, and IL-2 incorporated into a hydrogel lacking HA (supplemented collagen hydrogel) did not significantly increase IL-10 production over plate-bound activation by these agents (Figure 1(b)), which demonstrated the potentiating influence of HA on IL-10 production. The unique capability of HA to stimulate IL10 production by $\mathrm{T}$ cells is underscored by the observation that hydrogels made from other types of ECM, including basement membrane components (Matrigel) and fibrin, are not stimulatory in vitro [20].

3.2. Supplemented HA Hydrogels Promote IL-10 Production In Vivo. To evaluate whether supplemented HA hydrogels could be used to induce IL-10 production in vivo, the gels were populated with $3 \times 10^{6} \mathrm{CD} 4(+) / G F P-F o x P 3(-)$ T cells from CD45.2 mice and injected into the peritoneal cavities of CD45.1 mice. By use of the CD45.1 and CD45.2 allelic 


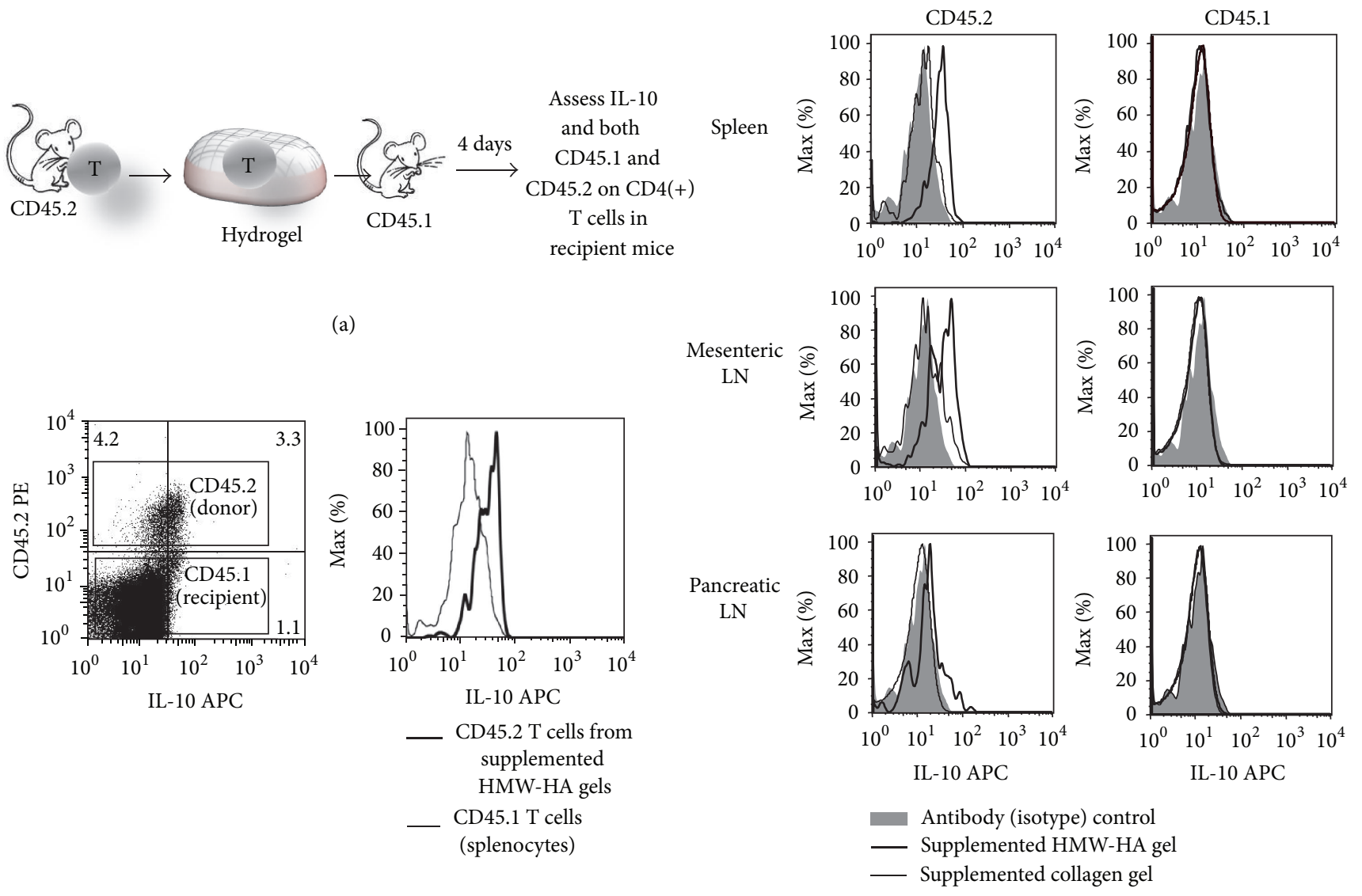

(b)

(c)

(d)

Figure 2: Supplemented HA hydrogels induce IL-10 in vivo. (a) Diagram of the experiment. Supplemented HA hydrogels, or control collagen hydrogels lacking HA, were populated with $3 \times 10^{6} \mathrm{CD} 4(+) /$ GFP-FoxP3(-)/CD45.2 donor cells and injected into the peritoneal cavities of CD45.1 recipient mice. Four days after implantation, lymphoid tissues were harvested, processed, and stained for intracellular IL-10 and CD markers. (b) Gating indicates relative IL-10 expression by CD45.2 donor and CD45.1 host T cells. (c) IL-10 expression by CD45.2 donor T cells harvested from HA hydrogel residue removed from the peritoneal cavity is substantially greater than that of CD 45.1 host T cells from the spleen. (d) IL-10 staining of CD3(+)/CD4(+) T cells harvested from the spleen and mesenteric/pancreatic lymph nodes (LN). The donor $\mathrm{T}$ cells from mice that received the supplemented HA hydrogels expressed higher levels of IL-10 than corresponding donor T cells from mice that received the control supplemented collagen hydrogels. Host T cells from these two groups of mice did not express IL-10 above levels of the nonspecific antibody (isotype) controls. In (c), and (d), data are representative of three experiments each.

markers, the donor and recipient cell populations could be distinguished. A schematic of this transfer protocol is shown in Figure 2(a). Four days after implantation, spleens and lymph nodes were harvested, processed, the released cells stained for intracellular IL-10 and CD markers, and gating performed to distinguish donor $\mathrm{T}$ cells from host $\mathrm{T}$ cells (Figure 2(b)). As controls, analogous supplemented collagen hydrogels lacking HA were populated with cells and injected into a designated set of mice.

After 4 days, a substantial volume of residual HA hydrogel was found within the peritoneal cavities of the treated mice; however, the control collagen hydrogels had dissolved. In separate experiments, we found that after 7 days no implanted HA hydrogels were identifiable, indicating that extensive catabolism of the hydrogels takes place in vivo.

The cells within the HA hydrogel residue 4 days after implantation were primarily CD45.2(+) and expressed IL10 at a high level relative to host $\mathrm{T}$ cells from the spleen
(Figure 2(c)). These cells remained FoxP3(-) (data not shown), consistent with our previous report that HA does not induce FoxP3 expression [19]. Cell isolates from the spleens and lymph nodes of the transplanted mice contained CD45.2(+) donor T cells (Figure 2(d)), which indicated that the $\mathrm{T}$ cells embedded in the hydrogels had migrated into lymphoid tissues. Donor $\mathrm{T}$ cells that migrated from the supplemented HA hydrogels expressed higher levels of IL10 than the corresponding donor T cells that migrated from the control collagen hydrogels. Host $\mathrm{T}$ cells from these two groups of mice did not express IL-10 above levels of the nonspecific antibody controls (Figure 2(d)). These data indicate that HA hydrogels providing endogenous TCR stimuli can be used as platforms to induce IL-10 production in vivo.

While supplemented HA hydrogels are a novel system for inducing implantable $\mathrm{T}$ cell populations that produce IL-10, we sought to devise an implantable platform that would elicit IL-10 production from endogenous $\mathrm{T}$ cells in 


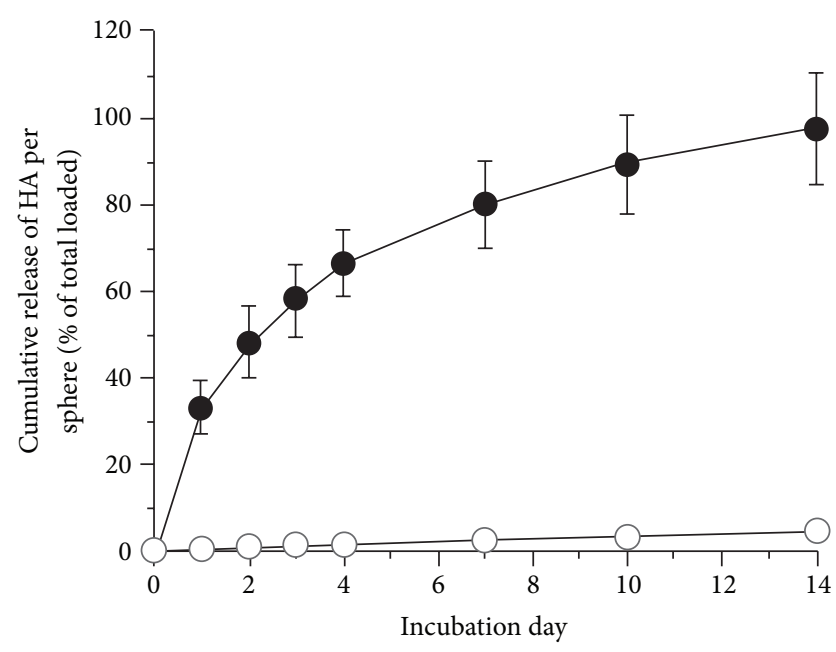

FIgURE 3: Kinetics of release of HA from alginate in vitro. Alginate spheres ( $2 \%$ alginate $/ 10 \mu \mathrm{L}$ volume $/ 2.2 \mathrm{~mm}$ diameter) incorporating $2.5 \mu \mathrm{g}$ of FITC-labeled $120 \mathrm{kDa}$ HA (closed circles) or FITC-labeled $1.5 \times 10^{6} \mathrm{Da} \mathrm{HA}$ (open circles) were cultured at $37^{\circ} \mathrm{C}$ in DPBS Ca/Mg, and the media collected at specific time points for measurement of released HA. Essentially all (98\%) of the $120 \mathrm{kDa}$ HA was released over 14 days, whereas less than $5 \%$ of the $1.5 \times 10^{6}$ Da HA was released during this time period. Error bars = standard deviations (error bars for the $1.5 \times 10^{6}$ Da group are within the diameter of the open circles). $n=10$ spheres for each group.

an antigen-specific manner. To this end, we adapted a novel bioengineered implant (BI) we had developed from an earlier study to combine the antigenic stimulus with sustained release of HA within the same construct.

3.3. Sustained Release of HA from BIs Induces IL-10 Production In Vivo. We recently reported on the development of the BI as a model system to explore improved approaches for islet transplantation [30]. The BI, sized for mesenteric or subcutaneous implantation in mice, consists of a disk-shaped PVA sponge infused with a type I collagen hydrogel that contains dispersed donor islets. To promote islet vascularization, the $\mathrm{BI}$ incorporates a spherical alginate construct for delivery of VEGF. Previously, we used syngeneic mice to demonstrate that BIs containing 450-500 islets and 20 ng of VEGF could reverse streptozotocin (STZ)-induced diabetes in $100 \%$ of recipients [30]. Notably, none of these mice required exogenous insulin therapy once the BIs began to fully regulate levels of blood glucose. Moreover, the transplanted mice responded to glucose challenge in a near-normal manner.

Induction of pro-tolerogenic cytokines, such as IL-10, is an appealing strategy to help facilitate transplantation of islets. Here, we have adapted our BI device to evaluate the capacity of HA in fluid form (i.e., HA not crosslinked to form a hydrogel) to elicit IL-10 production in an autoimmune setting. To test this model, we loaded the BI with islets expressing the OVA antigen, transferred in OVA-specific $\mathrm{T}$ cells, immunized the recipient mice with OVA, and asked whether these cells expressed IL-10 in an OVA or TCRspecific manner.
We first evaluated the kinetics of release of HA from $2 \mathrm{~mm}$ diameter, $2 \%$ alginate spheres under physiological conditions in vitro (Figure 3). We found that release of $1.5 \times 10^{6} \mathrm{Da}$ HA was linear, but relatively slow-less than $5 \%$ was released after 14 days. In contrast, the release of $120 \mathrm{kDa}$ HA was much more rapid-essentially $100 \%$ was released within 14 days, which was a useful time frame in which to analyze post-transplantation immune responses. We found that over $60 \%(63.7 \% \pm 6.1 \%)$ of the $120 \mathrm{kDa}$ HA loaded into each sphere was retained by the alginate after crosslinking with calcium. The BIs we designed for our experiments in vivo (Figure 4) incorporated a single alginate sphere containing $20 \mathrm{ng}$ of VEGF and 5 spheres that contained a total of $160 \mu \mathrm{g}$ of $120 \mathrm{kDa} \mathrm{HA}$ (the total is derived from a value of $32 \mu \mathrm{g}$ of HA per sphere, based on $64 \%$ retention of the $50 \mu \mathrm{g}$ of HA present in each sphere prior to calcium crosslinking). A set of control BIs incorporated one VEGF sphere and 5 spheres loaded with saline in place of the HA. The central hole of the BI was infused with a type I collagen hydrogel containing islets from RIPmOVA mice, which express chicken ovalbumin driven by the rat insulin promoter (RIP). BIs of this design were implanted into mesenteric pockets of RIPmOVA/Rag2 ${ }^{-/-}$mice (one BI per mouse), followed by injection of the mice 24 hours later with $1 \times 10^{5}$ purified OVA-specific CD4(+) DO11.10 T cells. Forty-eight hours after implantation, each mouse was immunized with $100 \mu \mathrm{g}$ of OVA peptide (aa 323-339). On day 14 after implantation, splenocytes were isolated from the mice and assayed for IL-10 production in vitro after 96 hours of stimulation with either anti-CD3/anti-CD28 antibodies or antigen-specific OVA peptide (Figure 5(a)). In this assay, the splenocytes from the mice treated with HA produced more IL-10 than the splenocytes from the control mice that were not exposed to HA. This differential response was observed when the splenocytes were given either a nonspecific stimulus with CD3/CD28 (Figure 5(b)) or a specific stimulus with OVA peptide (Figure 5(c)). Unfortunately, these data do not allow us to discern which cells are the source of IL-10 in this assay and specifically whether the cells in question are FoxP3(+) Tregs or FoxP3(-) conventional T cells.

\section{Discussion}

We demonstrate, using two separate model systems, that delivery of HA together with antigenic signals promotes the production of IL-10 in vivo. Our data suggest a potential clinical application for HA-mediated induction of IL-10producing $\mathrm{T}$ cells using injectable hydrogels. HA hydrogel platforms are in development for a variety of applications, including drug delivery and wound dressings, and are noted for their biocompatibility [31,32]. In the present study, we have shown that augmentation of HA hydrogels with a complex of biomolecules that provide TCR stimulation in addition to the HA signal can deliver the requisite cues for IL-10 induction, both in vitro and in vivo.

In treatments of diabetic patients that involve transplantation of islets, controlling rejection is typically accomplished by systemic immunosuppressive compounds. Dosing 


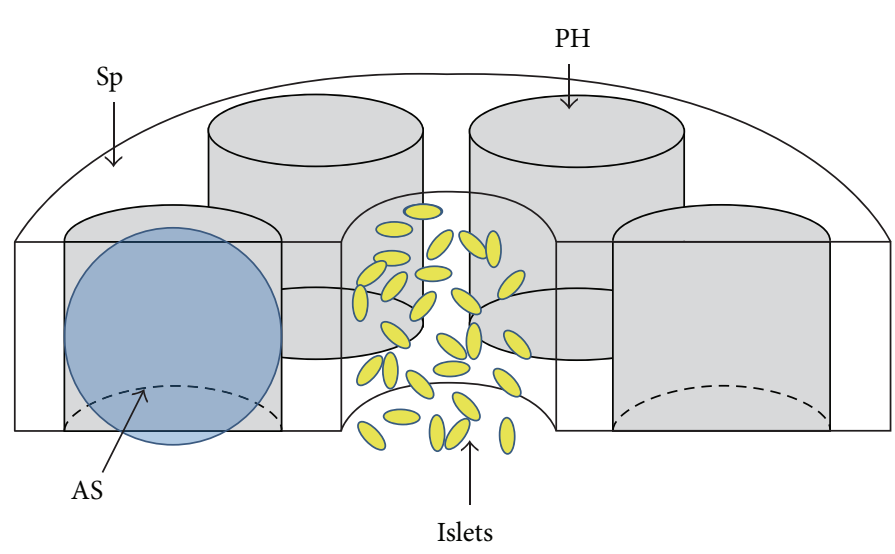

(a)

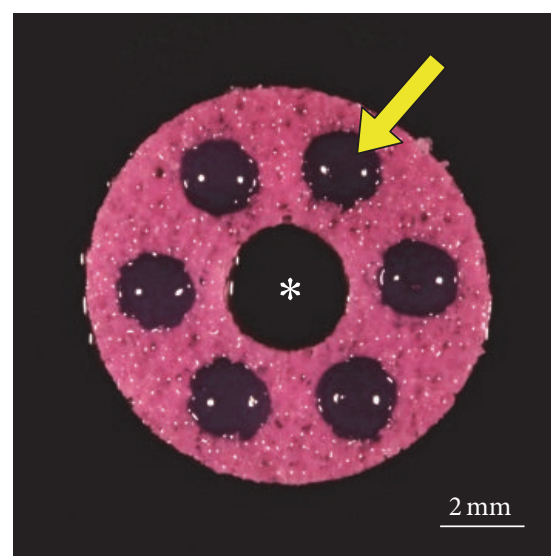

(b)

FIGURE 4: BI designed for evaluation of immune modulation by HA in vivo. (a) Cut-away diagram of the BI. A disk-shaped PVA sponge (Sp) scaffold provides mechanical support. Alginate spheres (AS-blue) occupy the six peripheral holes (PH) of the sponge. A central hole in the sponge contains islets (yellow) suspended in a type 1 collagen hydrogel (not shown). The collagen hydrogel also infuses the sponge. For clarity, the pores of the sponge are not depicted. (b) A PVA sponge scaffold (stained pink from culture medium) is oriented to show the empty central hole (asterisk) and the six peripheral holes, which each contain an alginate sphere (e.g., arrow). The scaffold is $10 \mathrm{~mm}$ in diameter $\times 2 \mathrm{~mm}$ thick.

of these compounds is a difficult balance-levels must be low enough to permit a reasonable degree of protective immunity against pathogenic organisms, but high enough to effectively suppress allo- and autoimmune activity. In the case of simultaneous pancreas-kidney (SPK) transplants, some current immunosuppression regimens are inadequate to control autoimmunity $[33,34]$. Moreover, no matter what the dose, systemic immunosuppression can be accompanied by a variety of undesirable side-effects on tissue and organ systems that are not directly associated with the transplant. In light of the problems associated with systemic treatments, an alternative approach would be to confine the delivery of immunotherapy to the implant itself. In this way, immunomodulatory compounds could be delivered at relatively high concentrations, but within the limited volume of the implant, thereby minimizing side-effects on tissues and organs outside the zone of delivery. To this end, the BI described here includes a mechanically-supportive scaffold and ECM hydrogel that concentrates the islets in a small volume, and a sustained-release component for local delivery of immunomodulatory compounds.

In the present study, we have adapted our BI to release HA in fluid form. Rather than using HA of $1-1.5 \times 10^{6} \mathrm{Da}$ that is typically incorporated into HA hydrogels, we used HA with a 10 -fold lower MW $(120 \mathrm{kDa})$ to provide release kinetics that were optimal for the 14-day duration of our experiments in vivo. Of note, we observed that this shorter HA could induce an IL-10 response from host mice. To our knowledge, this observation is the first demonstration of IL-10 production by $\mathrm{HA}$ of this weight class. It is possible that the $120 \mathrm{kDa} \mathrm{HA}$ is crosslinked into higher MW forms after its release into tissue from alginate, which could be accomplished by HA-binding molecules such as inter-alpha-trypsin inhibitor $(\mathrm{I} \alpha \mathrm{I})$ and/or tumor necrosis factor-stimulated gene-6 protein (TSG-6) which are present at sites of inflammation and which are known to crosslink HA into macromolecular assemblies [3537]. Such crosslinking could result not only in a functional increase in the MW of HA, but also promote the retention of $\mathrm{HA}$ in the fibrovascular tissue that invades the BI [30] and in the tissues immediately surrounding the implant. Our future studies will continue to use BIs as platforms to evaluate the effectiveness of HA and other specific ECM and cytokine environments on islet survival and reversal of diabetes in the setting of autoimmunity.
Abbreviations
HA:
BRI: $\quad$ Benaroya Research Institute;
BI: bioengineered implant;
GFP: $\quad$ green fluorescent protein;
IL-2, IL-10: interleukin-2, -10;
HS: heparan sulfate;
NMS: $\quad$ normal mouse serum;
TCR: $\quad$ T cell receptor complex;
Tregs: regulatory $\mathrm{T}$ cells;
VEGF: vascular endothelial growth factor.

\section{Conflicts of Interest}

The authors acknowledge no competing financial interests. Specifically, the authors have no conflict of interest with any trademark mentioned in this manuscript.

\section{Acknowledgments}

The authors wish to thank Drs. Susan Potter-Perigo and Thomas N. Wight for providing the FITC-HA used in our studies. This work was supported in part by National Institutes of Health grants R01 DK096087, R01 HL113294, and 


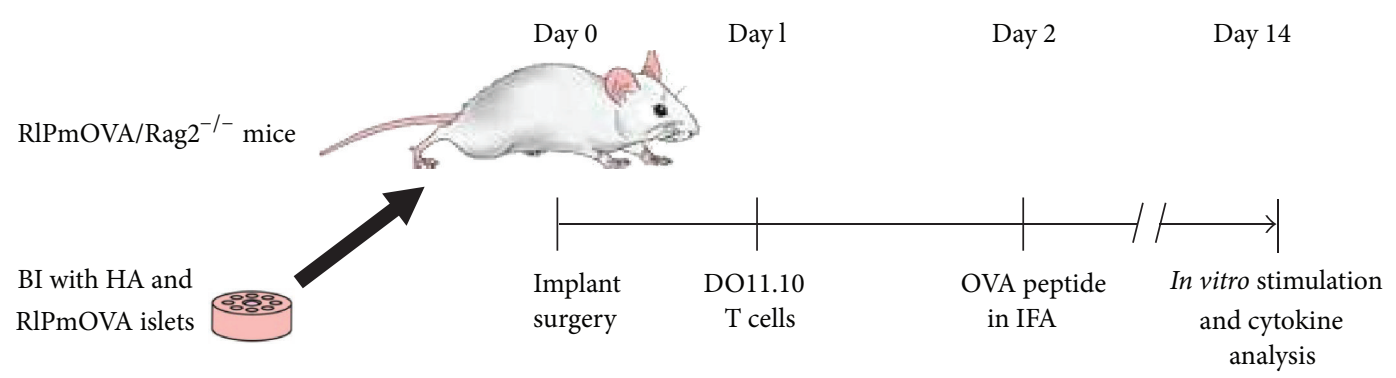

(a)

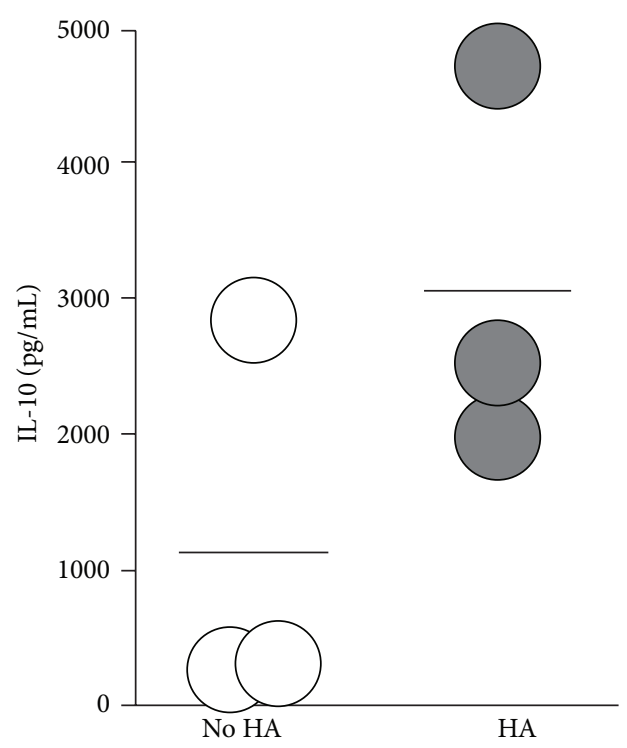

(b)

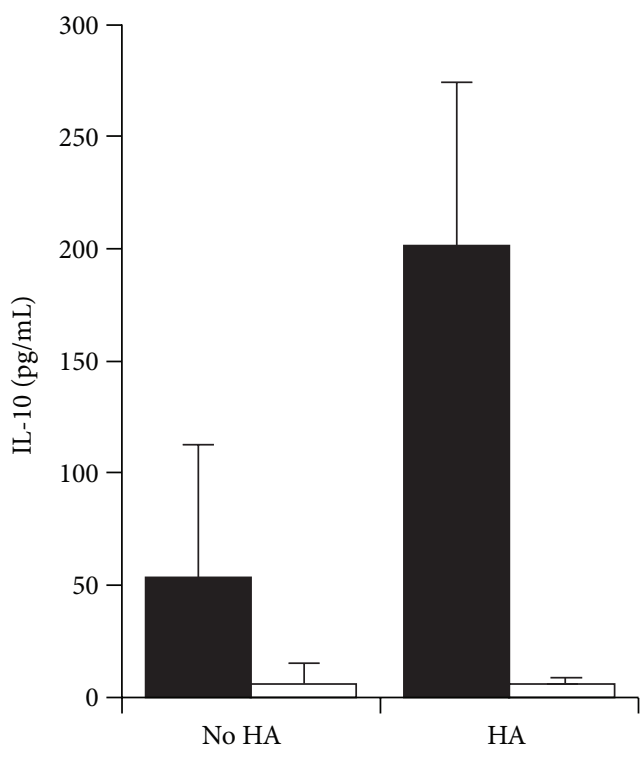

(c)

Figure 5: Local release of fluid HA from BIs generates IL-10 producing T cells in vivo. (a) Diagram of the experiment. BIs incorporating RIPmOVA islets and alginate spheres loaded with HA or saline (controls) were implanted in RIPmOVA / Rag2 $2^{-/-}$mice, followed by injection of OVA-specific DO11.10 T cells on day 1 and $100 \mu \mathrm{g}$ of OVA peptide in incomplete Freund's adjuvant (IFA) on day 2. On day 14, splenocytes were harvested and assayed for IL-10 production in response to antigenic stimulation in vitro. (b) IL-10 production by splenocytes from recipient animals in response to stimulation with anti-CD3/CD28 antibodies. (c) IL-10 production in response to OVA peptide (black bars) or no peptide (white bars). In (b) and (c), cells were stimulated for 96 hours, and culture supernates in contact with the stimulated cells for 72 hours were assayed by ELISA. Data are representative of 2 experiments totaling 5 mice in each group.

U01 AI101984 to Paul L. Bollyky; the Klorfine Foundation and Gilbertson Foundation (to Robert B. Vernon); and USAMRAA/DOD W81XWH-10-1-0149 and Washington State Life Sciences Discovery Fund Grant no. 4553677 to Gerald T. Nepom.

\section{References}

[1] C. Asseman, S. Mauze, M. W. Leach, R. L. Coffman, and F. Powrie, "An essential role for interleukin 10 in the function of regulatory T cells that inhibit intestinal inflammation," Journal of Experimental Medicine, vol. 190, no. 7, pp. 995-1004, 1999.

[2] E.-O. Glocker, D. Kotlarz, K. Boztug et al., "Inflammatory bowel disease and mutations affecting the interleukin-10 receptor," The New England Journal of Medicine, vol. 361, no. 21, pp. 2033-2045, 2009.

[3] M. G. Roncarolo, S. Gregori, M. Battaglia, R. Bacchetta, K. Fleischhauer, and M. K. Levings, "Interleukin-10-secreting type 1 regulatory $\mathrm{T}$ cells in rodents and humans," Immunological Reviews, vol. 212, pp. 28-50, 2006.
[4] K. W. Moore, M. R. de Waal, R. L. Coffman, and A. O'Garra, "Interleukin-10 and the interleukin-10 receptor," Annual Review of Immunology, vol. 19, pp. 683-765, 2001.

[5] A. M. VanBuskirk, W. J. Burlingham, E. Jankowska-Gan et al., "Human allograft acceptance is associated with immune regulation," Journal of Clinical Investigation, vol. 106, no. 1, pp. 145-155, 2000.

[6] S. Yi, M. Ji, J. Wu et al., "Adoptive transfer with in vitro expanded human regulatory $\mathrm{T}$ cells protects against porcine islet xenograft rejection via interleukin-10 in humanized mice," Diabetes, vol. 61, no. 5, pp. 1180-1191, 2012.

[7] K. S. Baker, M.-G. Roncarolo, C. Peters, M. Bigler, T. DeFor, and B. R. Blazar, "High spontaneous IL-10 production in unrelated bone marrow transplant recipients is associated with fewer transplant-related complications and early deaths," Bone Marrow Transplantation, vol. 23, no. 11, pp. 1123-1129, 1999.

[8] V. Daniel, C. Naujokat, M. Sadeghi, M. Wiesel, O. Hergesell, and G. Opelz, "Association of circulating interleukin (IL)-12- and IL10 -producing dendritic cells with time posttransplant, dose of 
immunosuppression, and plasma cytokines in renal-transplant recipients," Transplantation, vol. 79, no. 11, pp. 1498-1506, 2005.

[9] Y. C. Zhang, A. Pileggi, A. Agarwal et al., "Adeno-associated virus-mediated IL-10 gene therapy inhibits diabetes recurrence in syngeneic islet cell transplantation of NOD mice," Diabetes, vol. 52, no. 3, pp. 708-716, 2003.

[10] Y.-H. Kim, D.-G. Lim, Y.-M. Wee et al., "Viral IL-10 gene transfer prolongs rat islet allograft survival," Cell Transplantation, vol. 17, no. 6, pp. 609-618, 2008.

[11] A. Rabinovitch, W. L. Suarez-Pinzon, O. Sorensen, R. V. Rajotte, and R. F. Power, "Combination therapy with cyclosporine and interleukin-4 or interleukin- 10 prolongs survival of syngeneic pancreatic islet grafts in nonobese diabetic mice: islet graft survival does not correlate with mRNA levels of type 1 or type 2 cytokines, or transforming growth factor- $\beta$ in the islet grafts," Transplantation, vol. 64, no. 11, pp. 1525-1531, 1997.

[12] M. Battaglia, A. Stabilini, E. Draghici et al., "Rapamycin and interleukin-10 treatment induces T regulatory type 1 cells that mediate antigen-specific transplantation tolerance," Diabetes, vol. 55, no. 1, pp. 40-49, 2006.

[13] N. Gagliani, S. Gregori, T. Jofra et al., "Rapamycin combined with anti-CD45RB mAB and IL-10 or with G-CSF induces tolerance in a stringent mouse model of islet transplantation," PloS One, vol. 6, no. 12, article e28434, 2011.

[14] Y. C. Zhang, A. Pileggi, R. D. Molano et al., "Systemic overexpression of interleukin-10 fails to protect allogeneic islet transplants in nonobese diabetic mice," Transplantation, vol. 80, no. 4, pp. 530-533, 2005.

[15] T. C. Laurent and J. R. E. Fraser, "Hyaluronan," The FASEB Journal, vol. 6, no. 7, pp. 2397-2404, 1992.

[16] D. Jiang, J. Liang, J. Fan et al., "Regulation of lung injury and repair by Toll-like receptors and hyaluronan," Nature Medicine, vol. 11, no. 11, pp. 1173-1179, 2005.

[17] C. Termeer, F. Benedix, J. Sleeman et al., "Oligosaccharides of hyaluronan activate dendritic cells via Toll-like receptor 4," Journal of Experimental Medicine, vol. 195, no. 1, pp. 99-111, 2002.

[18] J. D. Powell and M. R. Horton, “Threat matrix: low-molecularweight hyaluronan (HA) as a danger signal," Immunologic Research, vol. 31, no. 3, pp. 207-218, 2005.

[19] P. L. Bollyky, B. A. Falk, S. A. Long et al., "CD44 costimulation promotes FoxP3+ regulatory T cell persistence and function via production of IL-2, IL-10, and TGF- $\beta$," Journal of Immunology, vol. 183, no. 4, pp. 2232-2241, 2009.

[20] P. L. Bollyky, R. P. Wu, B. A. Falk et al., "ECM components guide IL-10 producing regulatory T-cell (TR1) induction from effector memory T-cell precursors," Proceedings of the National Academy of Sciences of the United States of America, vol. 108, no. 19, pp. 7938-7943, 2011.

[21] T.-L. Huang, H.-C. Hsu, K.-C. Yang, and F.-H. Lin, "Hyaluronan up-regulates IL-10 expression in fibroblast-like synoviocytes from patients with tibia plateau fracture," Journal of Orthopaedic Research, vol. 29, no. 4, pp. 495-500, 2011.

[22] A. Asari, T. Kanemitsu, and H. Kurihara, "Oral administration of high molecular weight hyaluronan $(900 \mathrm{kDa})$ controls immune system via toll-like receptor 4 in the intestinal epithelium," Journal of Biological Chemistry, vol. 285, no. 32, pp. 2475124758, 2010.

[23] A. Gigante and L. Callegari, "The role of intra-articular hyaluronan (Sinovial) in the treatment of osteoarthritis," Rheumatology International, vol. 31, no. 4, pp. 427-444, 2011.
[24] Y. Kim, Y.-S. Lee, J.-H. Hahn et al., "Hyaluronic acid targets CD44 and inhibits FceRI signaling involving PKC $\delta$, Racl, ROS, and MAPK to exert anti-allergic effect," Molecular Immunology, vol. 45, no. 9, pp. 2537-2547, 2008.

[25] Q. Zeng, Z. Yu, J. You, and Q. Zhang, "Efficacy and safety of seprafilm for preventing postoperative abdominal adhesion: systematic review and meta-analysis," World Journal of Surgery, vol. 31, no. 11, pp. 2125-2131, 2007.

[26] G. Kogan, L. Šoltés, R. Stern, and P. Gemeiner, "Hyaluronic acid: a natural biopolymer with a broad range of biomedical and industrial applications," Biotechnology Letters, vol. 29, no. 1, pp. 17-25, 2007.

[27] R. D. Price, V. Das-Gupta, I. M. Leigh, and H. A. Navsaria, "A comparison of tissue-engineered hyaluronic acid dermal matrices in a human wound model," Tissue Engineering, vol. 12, no. 10, pp. 2985-2995, 2006.

[28] G. Gravante, R. Sorge, A. Merone et al., "Hyalomatrix PA in burn care practice: results from a national retrospective survey, 2005 to 2006," Annals of Plastic Surgery, vol. 64, no. 1, pp. 69-79, 2010.

[29] G. D. Prestwich, X. Z. Shu, Y. Liu et al., "Injectable synthetic extracellular matrices for tissue engineering and repair," Advances in Experimental Medicine and Biology, vol. 585, pp. 125-133, 2006.

[30] R. B. Vernon, A. Preisinger, M. D. Gooden et al., "Reversal of diabetes in mice with a bioengineered islet implant incorporating a type I collagen hydrogel and sustained release of vascular endothelial growth factor," Cell Transplantation, vol. 21, no. 10, pp. 2099-2110, 2012.

[31] M. A. Serban, A. Scott, and G. D. Prestwich, "Use of hyaluronanderived hydrogels for three-dimensional cell culture and tumor xenografts," Current Protocols in Cell Biology, chapter 10, unit $10.14,2008$.

[32] K. R. Kirker, Y. Luo, J. H. Nielson, J. Shelby, and G. D. Prestwich, "Glycosaminoglycan hydrogel films as bio-interactive dressings for wound healing," Biomaterials, vol. 23, no. 17, pp. 3661-3671, 2002.

[33] E. Laughlin, G. Burke, A. Pugliese, B. Falk, and G. Nepom, "Recurrence of autoreactive antigen-specific CD4+ T cells in autoimmune diabetes after pancreas transplantation," Clinical Immunology, vol. 128, pp. 23-30, 2008.

[34] F. Vendrame, A. Pileggi, E. Laughlin et al., "Recurrence of type 1 diabetes after simultaneous pancreas-kidney transplantation, despite immunosuppression, is associated with autoantibodies and pathogenic autoreactive CD4 T-cells," Diabetes, vol. 59, pp. 947-957, 2010.

[35] M. S. Rugg, A. C. Willis, D. Mukhopadhyay et al., "Characterization of complexes formed between TSG- 6 and inter- $\alpha$-inhibitor that act as intermediates in the covalent transfer of heavy chains onto hyaluronan," Journal of Biological Chemistry, vol. 280, no. 27, pp. 25674-25686, 2005.

[36] L. Zhuo and K. Kimata, "Structure and function of inter- $\alpha$ trypsin inhibitor heavy chains," Connective Tissue Research, vol. 49, no. 5, pp. 311-320, 2008.

[37] D. Mukhopadhyay, A. Asari, M. S. Rugg, A. J. Day, and C. Fülöp, "Specificity of the tumor necrosis factor-induced protein 6-mediated heavy chain transfer from inter- $\alpha$-trypsin inhibitor to hyaluronan: implications for the assembly of the cumulus extracellular matrix," Journal of Biological Chemistry, vol. 279, no. 12, pp. 11119-11128, 2004. 


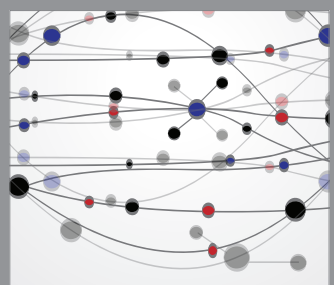

The Scientific World Journal
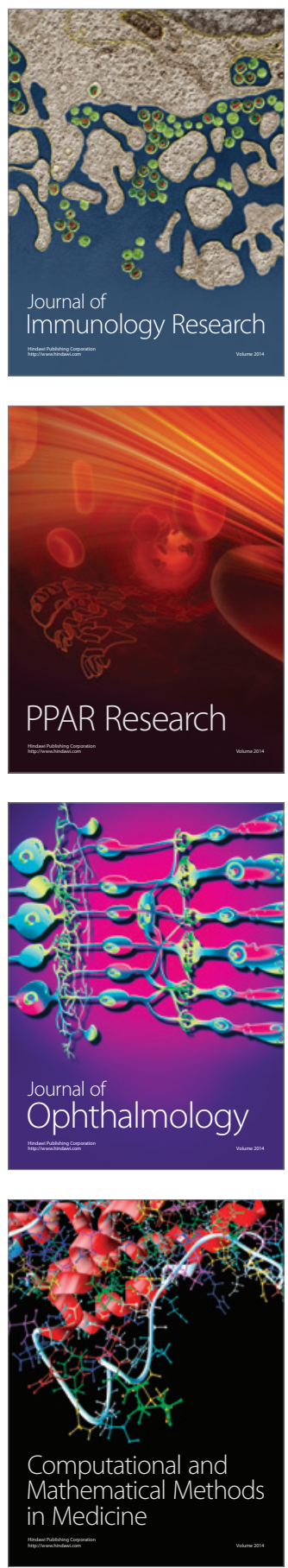

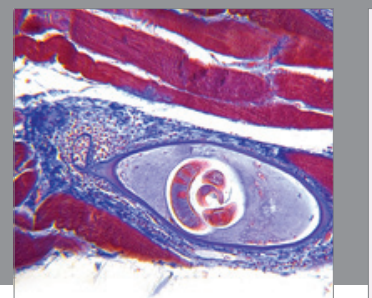

Gastroenterology

Research and Practice
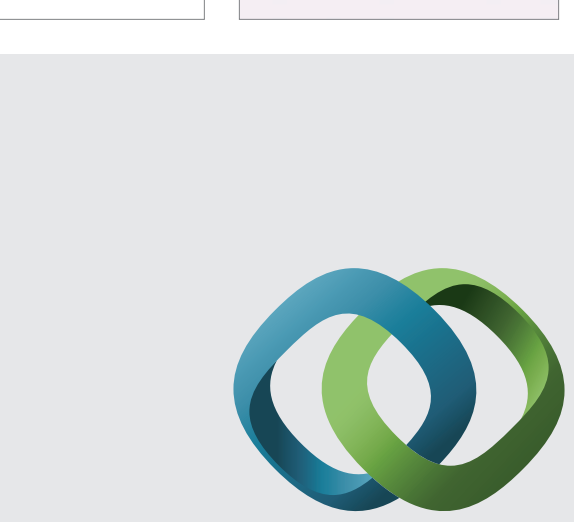

\section{Hindawi}

Submit your manuscripts at

http://www.hindawi.com
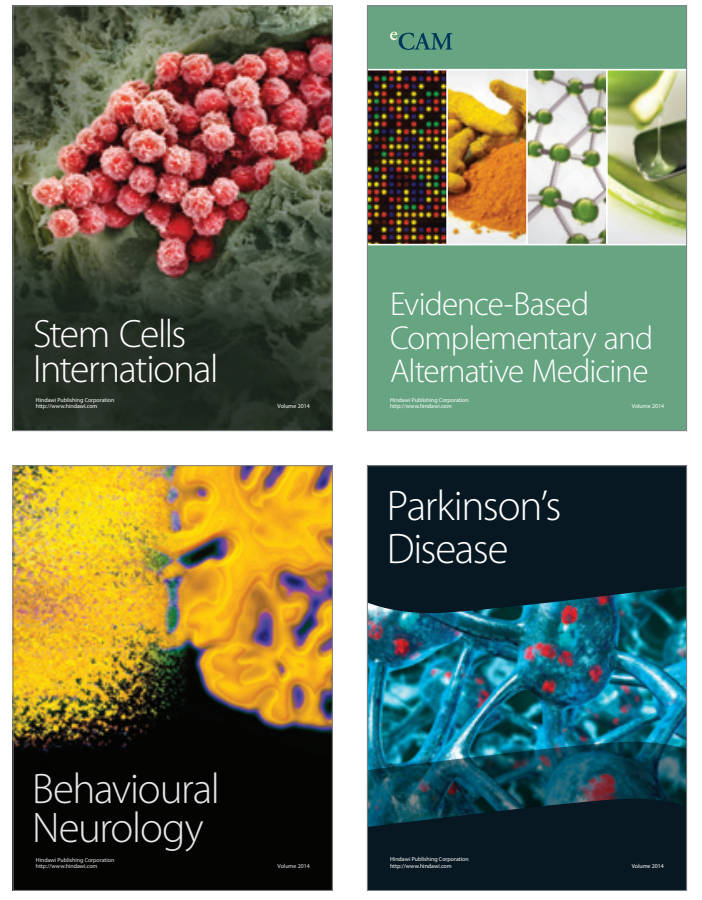
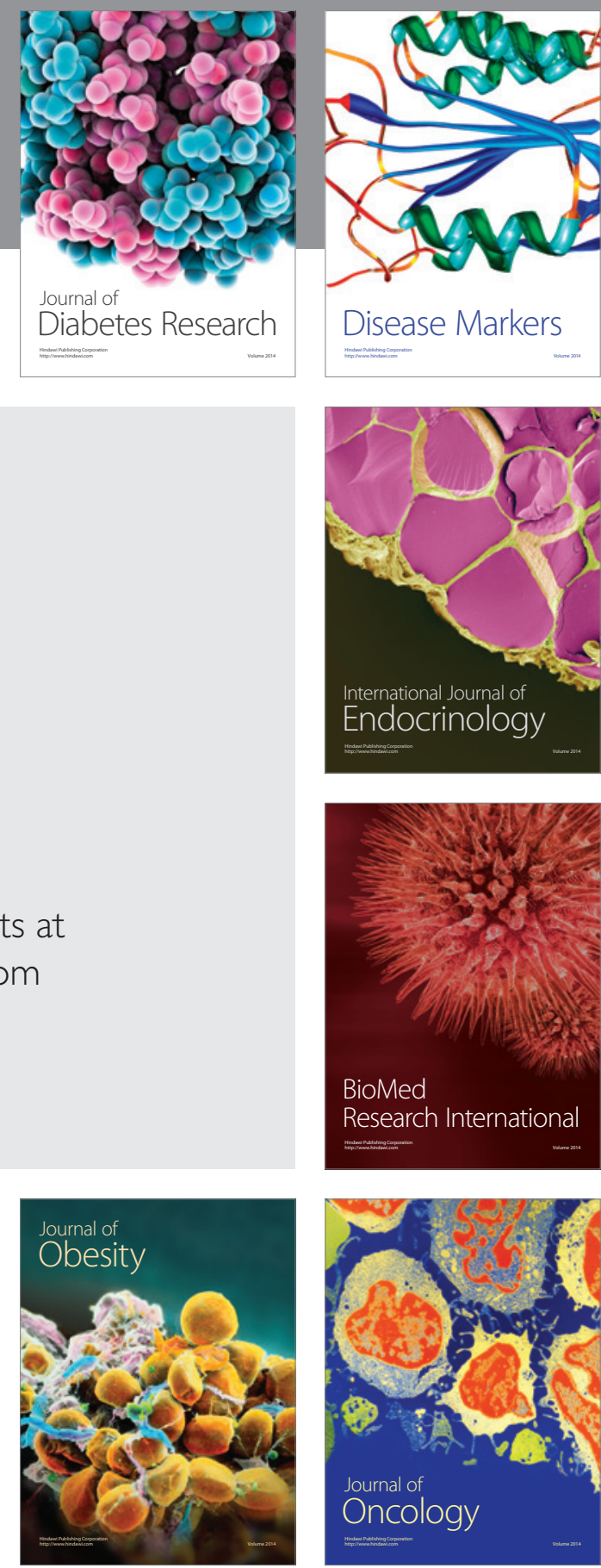

Disease Markers
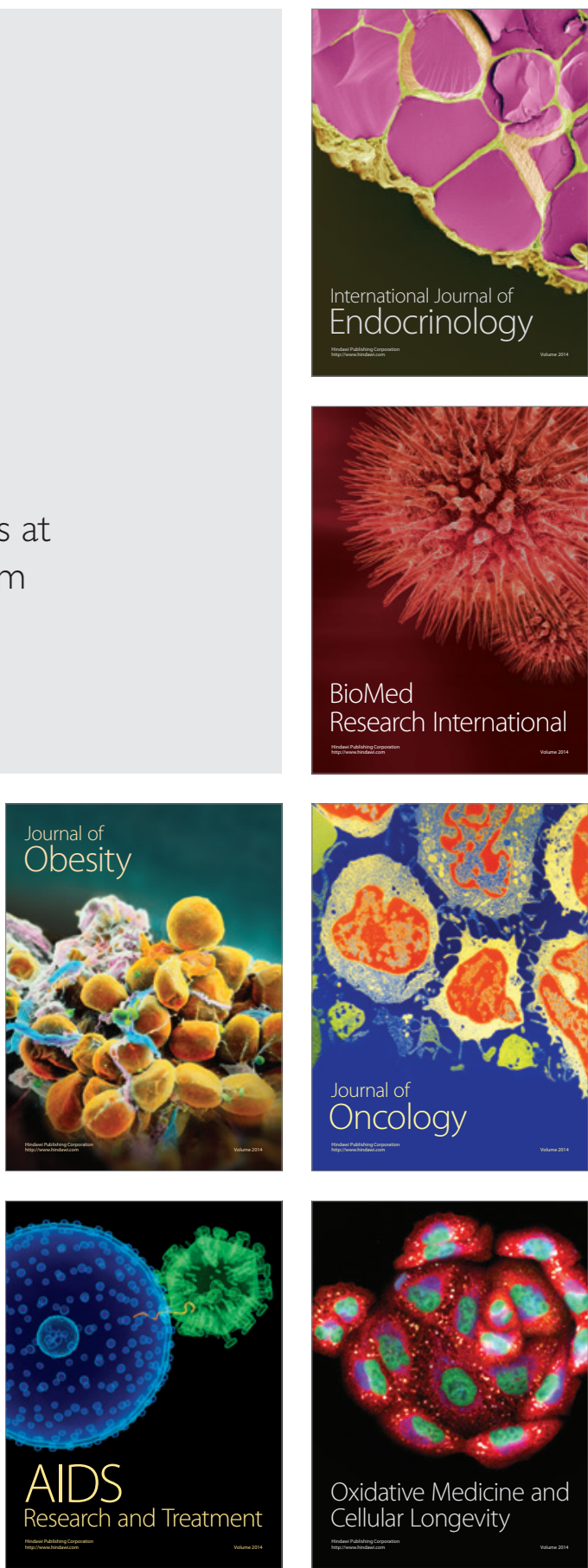